\title{
Targeted Next Generation Sequencing improves detection and quantification of rare species from eDNA
}

\author{
Kristen M Westfall ${ }^{\ddagger}$, Thomas W Therriault ${ }^{\ddagger}$, Cathryn L Abbott ${ }^{\ddagger}$ \\ ‡ Fisheries and Oceans Canada, Nanaimo, Canada
}

Corresponding author: Cathryn L Abbott (cathryn.abbott@dfo-mpo.gc.ca)

Received: 25 Feb 2021 | Published: 04 Mar 2021

Citation: Westfall KM, Therriault TW, Abbott CL (2021) Targeted Next Generation Sequencing improves detection and quantification of rare species from eDNA. ARPHA Conference Abstracts 4: e65075. https://doi.org/10.3897/aca.4.e65075

\begin{abstract}
Targeted species detection from eDNA is central to identifying and quantifying rare (i.e. invasive or endangered) species to inform conservation and resource management. Here we introduce a new targeted Next Generation Sequencing (tNGS) assay that shows improved detection relative to quantitative (q)PCR at low eDNA concentrations and increased precision to detect spatial variation in eDNA concentration related to species abundance. We compare the tNGS and qPCR methods using invasive European green crab (Carcinus maenas) in the northeast Pacific Ocean as a test case, and find that crab abundance measured by traditional trapping is significantly correlated with eDNA concentration across multiple sites for both methods. However, the tNGS assay outperformed qPCR in all tests: (1) increased precision of eDNA concentration estimation; (2) a $7-10 \%$ increase in detection probability at low abundance sites; and (3) greater power to detect spatial variation in eDNA concentration. The accuracy of predicting green crab abundance from eDNA concentration increased with the number of field replicates sampled and did not change appreciably over a tidal cycle. Green crab eDNA concentration behaving similarly to abundance measured from trapping demonstrates great promise for this tool to be implemented for early detection and routine monitoring surveys. The tNGS assay is easily accessible for surveying other species with existing qPCR assays and can
\end{abstract}


thus be potentially important for detection and quantification of any species of high interest to management.

\section{Keywords}

environmental DNA, invasive species, next generation sequencing, qPCR, survey method, green crab

\section{Presenting author}

Kristen M. Westfall

\section{Presented at}

1st DNAQUA International Conference (March 9-11, 2021) 